\title{
approche pluridisciplinaire des problèmes posés par un glissement de terrain sur faible pente
}

\author{
par \\ A. Giraud \\ I.R.I.G.M. (Université de Grenoble) \\ J.P. Gourc \\ I.R.I.G.M. (Université de Grenoble) \\ L. Besson \\ Direction Départementale de l'Agriculture de I'Isère \\ D. Fabre \\ I. R. I.G.M. (Université de Grenoble)
}

\section{Généralités}

Le glissement étudié s'est déclenché le 9 avril 1978 sur la commune de Monestier du Percy (Isère) au lieu-dit serre des Bayles. Des mouvements importants se sont encore produits ensuite pendant quatre jours, affectant une zone de pâturages de pente faible, d'une superficie totale de 9 hectares.

L'observation des photographies aériennes antérieures (1956 et 1970) montre qu'il existait déjà à ces époques des indices de mouvement assez nets (petites niches d'arrachement fraiches, bourrelets...) surtout dans la partie NE. Cette zone n'a été que peu réactivée en 1978, les mouvements les plus spectaculaires se produisant surtout dans la partie SW sur une superficie d'environ 5 hectares.

\subsection{Contexte géologique}

Les "terres noires" du substratum de cette région (épaisse série de schistes sombres du Callovien et de l'Oxfordien) affleurent à l'amont et à l'aval du glissement dans le lit du torrent du Chapolet. Ces schistes sont pentés de $40^{\circ}$ vers le SW alors que la vallée est orientée N30E et que la pente glissée (rive droite du torrent) plonge globalement vers le NW.

Sur ce substratum rocheux reposent des argiles glaciolacustres à litage horizontal qui présentent une alternance régulière de lits clairs silteux et de lits argileux plus sombres (épaisseur comprise entre 1 et $20 \mathrm{~mm}$ ). Elles correspondent au remplissage d'un lac de barrage glaciaire datant de l'extension maximale du glacier de I'Isère au Würm II (fig. 1). Leur épaisseur qui peut atteindre 200 mètres ne doit pas dépasser quarante mètres à l'endroit du glissement, si l'on se réfère à la carte géologique à l'échelle du $1 / 2000$ (fig. 3).

Enfin, on trouve, surmontant les argiles, une couche de un à deux mètres de formations alluviales et torrentielles, datées elles aussi du Würm.

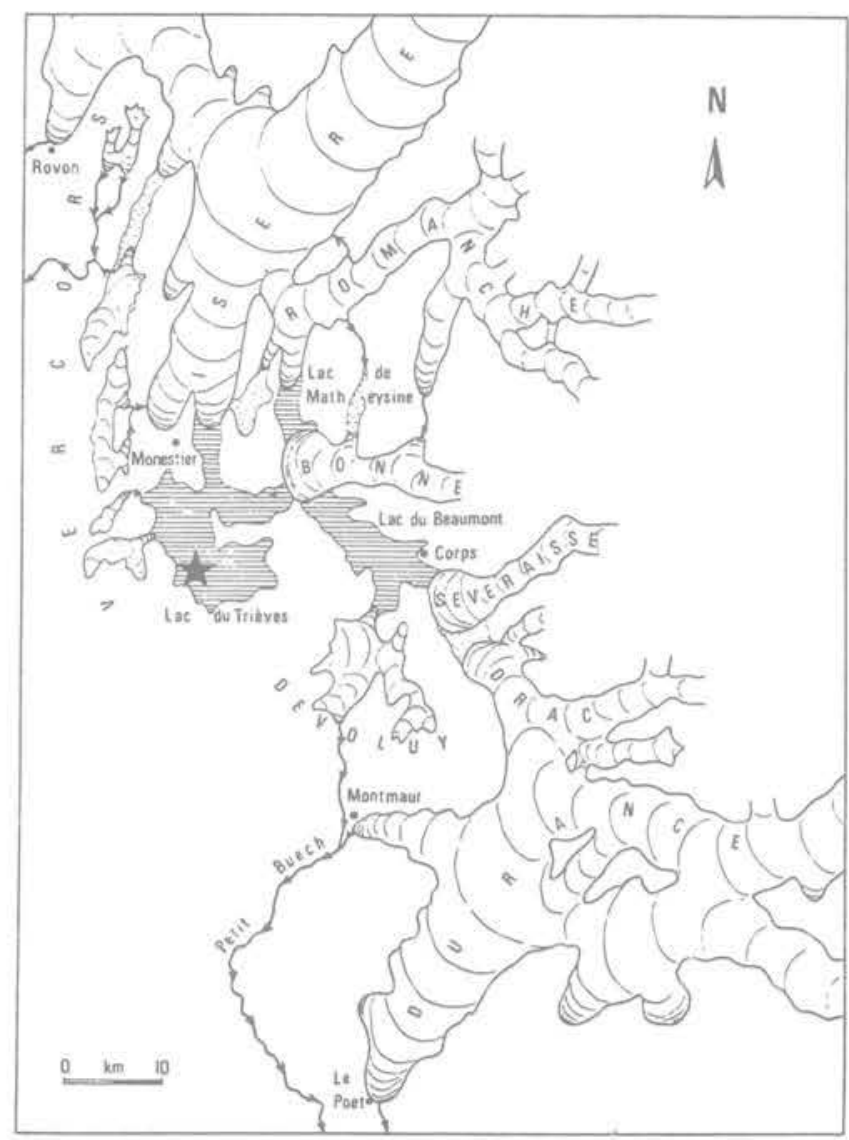

Fig. 1 Extension des glaciers au maximum du Würm et localisation du lac de barrage glaciaire du Trièves (d'après Monjuvent, 1973). Le secteur étudié est indiqué par une étoile 


\subsection{Conditions hydrologiques et climatiques}

Avant le glissement, en amont de la zone instable, existait une zone marécageuse assez vaste dont les eaux devaient contribuer à l'alimentation de suintements observables dans la pente sur les photographies aériennes de 1956 et 1970 .

Les données météorologiques analysées (fig. 2) montrent que la pluviométrie (surtout sous forme de neige) avait été forte pendant les deux mois consécutifs (203 mm en Février 1978 et $197 \mathrm{~mm}$ en Mars 1978) qui ont précédé le glissement. Après la fonte très rapide du manteau neigeux, liée à une période de dégel, le glissement s'est produit sous l'effet de l'augmentation des pressions interstitielles.

\section{Description géologique du glissement}

\subsection{Cartographie}

Une cartographie détaillée du glissement a été effectuée à l'échelle du 1/2000 à partir d'un orthophotoplan (fig. 3). Elle nous a permis de mettre en place certains détails importants (fissures principales, zones de rétention d'eau, lames d'argile apparentes...), de reporter la direction et le pendage du litage des varves après glissement et de situer avec précision les profils géologiques et les prélèvements d'échantillons.

Au niveau de la niche d'arrachement, nous trouvons sous vingt centimètres de terre végétale, une épaisseur de un mètre cinquante à deux mètres d'alluvions du Würm. Au contact des alluvions et des argiles varvées se produisent des suintements d'eau qui altèrent sur une vingtaine de centimètres le sommet des argiles.

Dans la partie supérieure du glissement comprise entre la niche d'arrachement et les restes du chemin, le litage est penté de 15 à $40^{\circ}$ vers le NW. L'orientation du litage semble donc évoquer à ce niveau un glissement des blocs d'argile dans la pente.

Par contre, la surface du chemin et le litage des argiles dans tout le secteur situé en contrebas du chemin sont pentés de 20 à $40^{\circ}$ vers le SE. La disposition de ce litage, l'aspect général de toute cette zone (basculement de blocs, effondrement locaux) suggèrent que toute la partie amont du glissement a été sujette à d'importantes distorsions. La partie basse de la zone déstabilisée semble avoir subi un écoulement plan généralisé avec des translations horizontales importantes (de l'ordre de $70 \mathrm{~m}$ en certains points). Enfin, en pied de glissement, une coulée a comblé le lit du ruisseau du Chapolet et en a décalé le cours d'une trentaine de mètres vers le NW. Un petit lac atteignant dix mètres de profondeụr s'est formé derrière ce barrage naturel.

\subsection{Recherche de la surface de rupture par géophysique}

En l'absence de forage, plusieurs sondages sismiques ont été réalisés. Les résultats de cette campagne apportent quelques indications sur l'épaisseur des terrains remaniés. Ils sont cependant insuffisants pour tracer avec précision la surface de rupture la plus probable.

- Dans les argiles variées non remaniées (sondages d'étalonnage I réalisés en amont de la niche d'arrachement) les vitesses mesurées sont comprises entre 1500 et $2000 \mathrm{~m} / \mathrm{s}$.

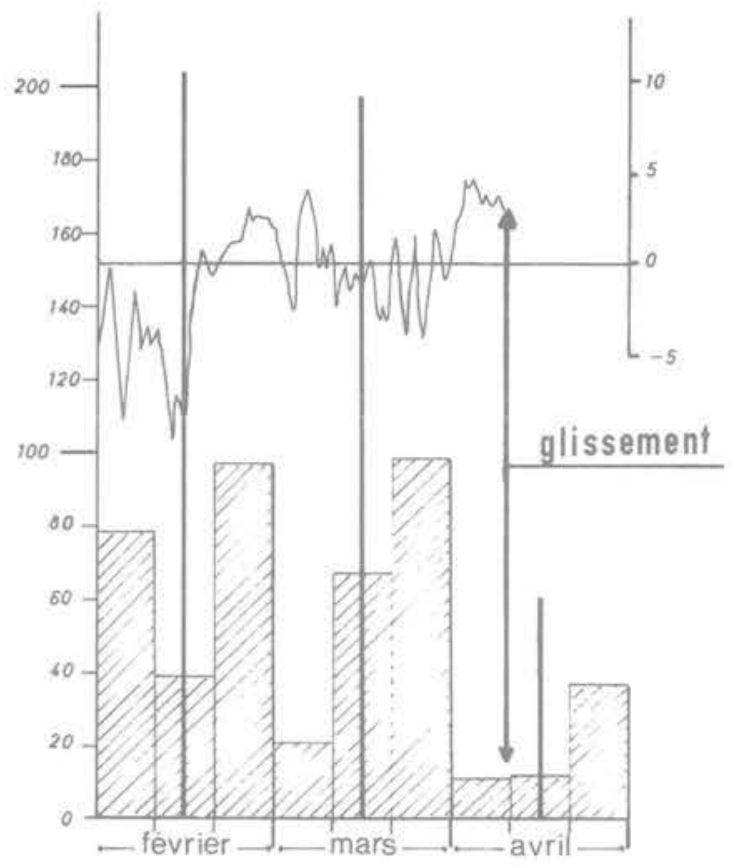

$$
\begin{gathered}
\text { hauteur des } \\
\text { précipitations } \\
\text { par décade }
\end{gathered}
$$

hauteur totale mensuelle des précipitations
Fig. 2 Diagramme des précipitations et des températures au printemps 1978 (stations météorologiques de Lalley et de Mens, Isère)

- Le profil III réalise selon une horizontale dans le corps du glissement est à peu près symétrique. II donne pour le terrain remanié une vitesse de $1300 \mathrm{~m} / \mathrm{s}$ sur une épaisseur de six à huit mètres.

- Le profil en long II (fig. 4) est au contraire nettement dissymétrique. L'interprétation conduit à un schéma de réfracteur incliné d'environ $12^{\circ}$ par rapport à la surface, soit $4^{\circ}$ de contre-pente par rapport à l'horizontale. Dans la partie centrale du glissement la profondeur de la zone remaniée est d'environ 20 mètres. Les résultats de ces sondages ne permettent pas de dire où se trouve exactement le contact entre les argiles varvées et les terres noires. En effet, d'après les études menées dans la région sur le tracé voisin de la RN 75 (voir Kosseoglu 1977), les terres noires saines correspondraient à une vitesse comprise entre 2600 et $3500 \mathrm{~m} / \mathrm{s}$, mais l'altération peut abaisser ces valeurs en dessous de $2000 \mathrm{~m} / \mathrm{s}$, ce qui introduit un risque de confusion avec les argiles varvées non remaniées.

\subsection{Identification géologique des argiles varvées}

Quelques essais d'identification ont été effectués sur les matériaux argileux du glissement. 


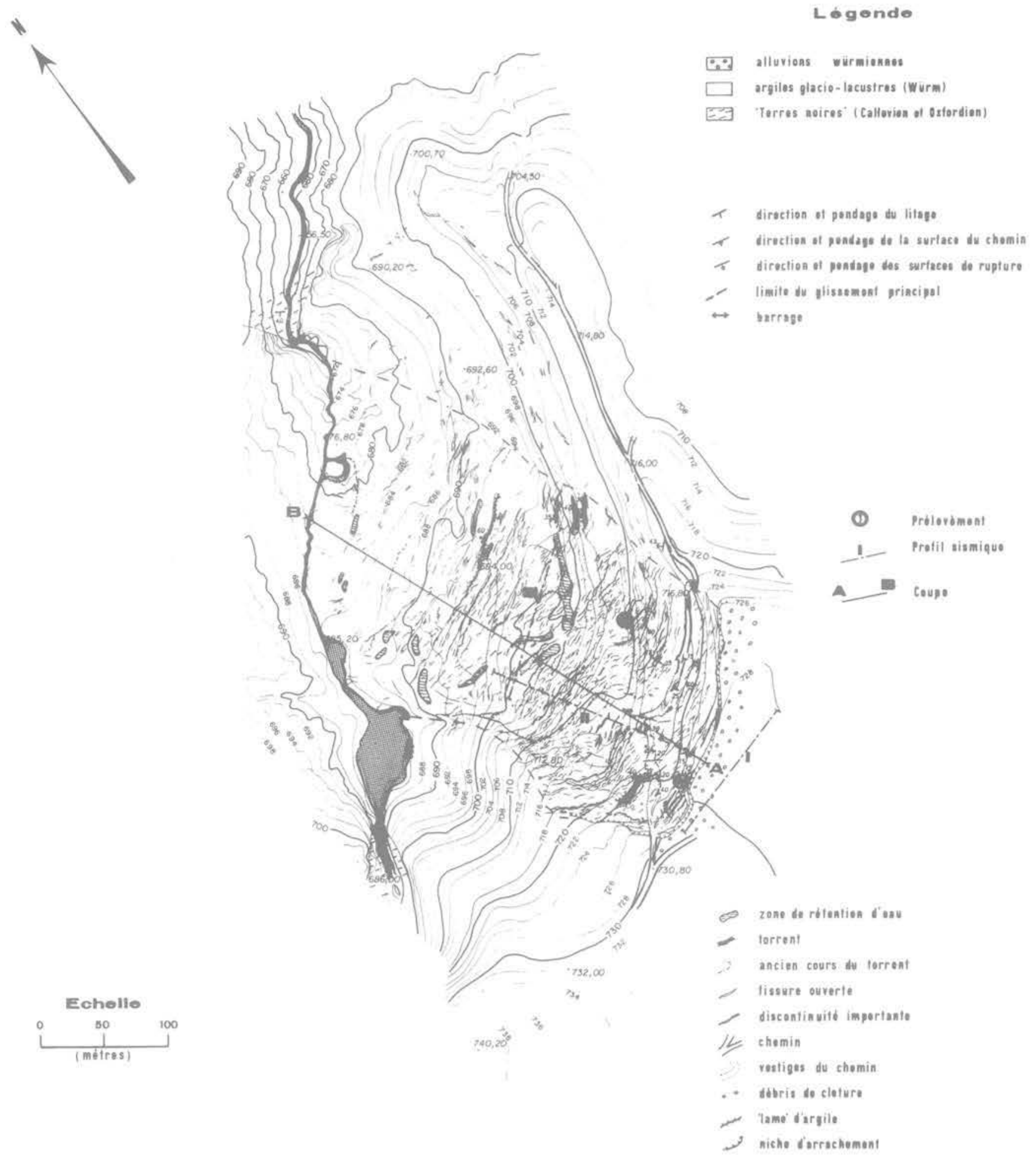

Fig. 3 Glissement de Monestier-du-Percy (/sère)

- La granulométrie indique une proportion d'argile assez forte dans les niveaux sombres $(60 \%<2 \mu)$ légèrement plus faible dans les niveaux clairs $(40 \%<2 \mu)$. Avec une limite de liquidité de $38 \%$ et un indice de plasticité de 17 , le matériau argileux sombre se classe parmi les argiles inorganiques de moyenne plasticité dans l'abaque de Casagrande.

- Des analyses chimiques par calcimétrie et diffraction aux rayons $X$ montre que la calcite prédomine largement dans les strates claires alors que les strates sombres comportent à parts à peu près égales la calcite, le quartz, l'illite et la chlorite.

\begin{tabular}{l|r|r}
\hline \multicolumn{1}{c|}{ Nature des minéraux } & $\begin{array}{r}\text { Strate } \\
\text { claire }\end{array}$ & $\begin{array}{c}\text { Strate } \\
\text { sombre }\end{array}$ \\
\hline Calcite & $50 \%$ & $25 \%$ \\
Quartz & $10 \%$ & $20 \%$ \\
Feldspaths & - & $5 \%$ \\
Illite & $15 \%$ & $10 \%$ \\
Chlorite & $6 \%$ & $10 \%$ \\
Kaolinite & $6 \%$ & $5 \%$ \\
Montmorillonite +I.M.C. & $12 \%$ & $5 \%$ \\
\hline
\end{tabular}

Tableau 1 Composition minéralogique des niveaux clairs et sombres 


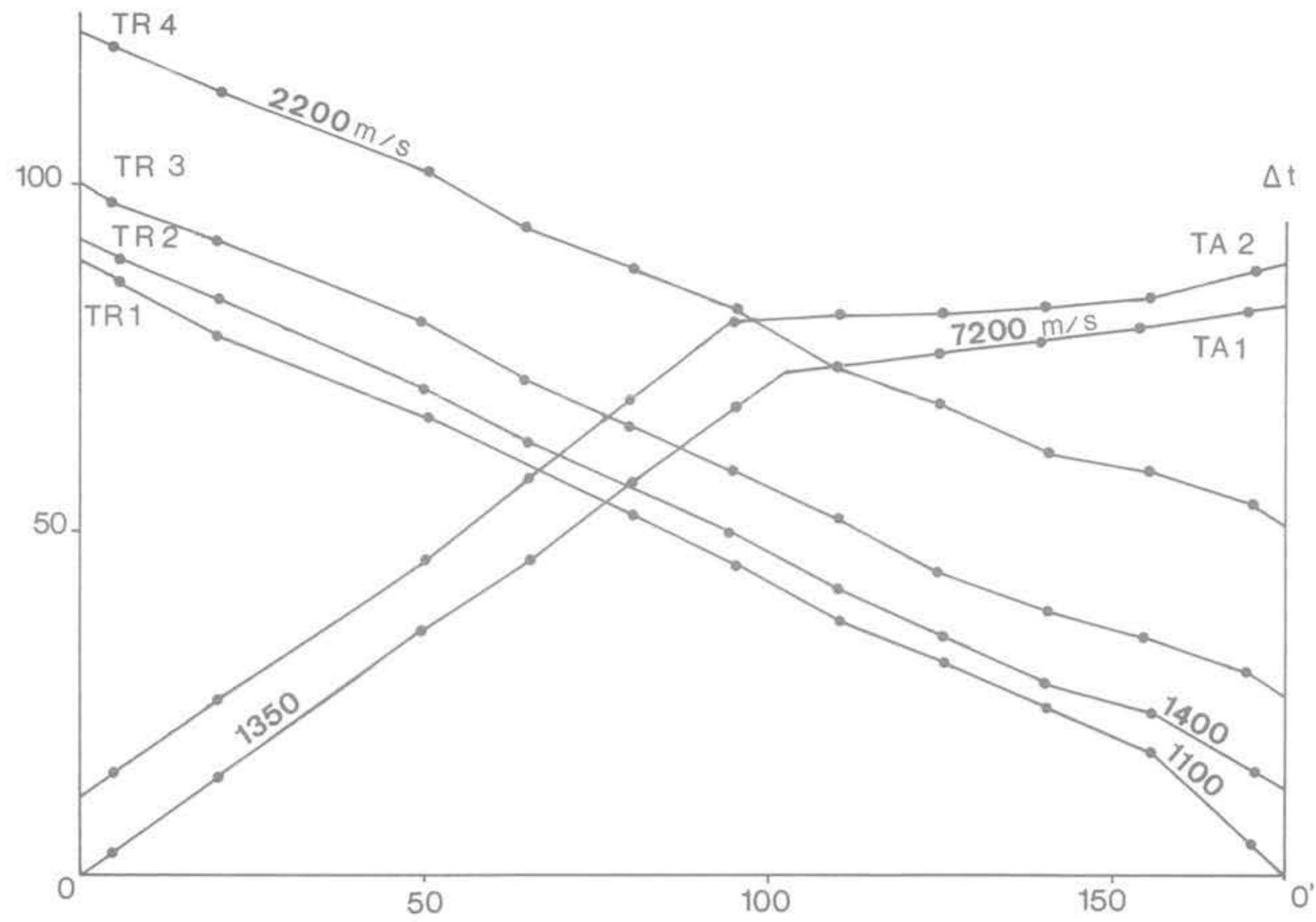

Fig. 4 Profil sismique $n^{\circ} / /$ réalisé parallèlement à la direction du glissement. La vitesse apparente de $7200 \mathrm{~m} / \mathrm{s}$ s'explique par l'inclinaison de l'interface (zone glissée - roche en place) par rapport à la surface topographique

Dans la fraction inférieure à $2 \mu$, on ne trouve, dans les deux cas, que des minéraux argileux (avec une fraction non négligeable de montmorillonite pour les strates claires).

L'interprétation de l'origine de ces argiles et de leur disposition en varves fait actuellement l'objet d'études complémentaires (comparaison avec la minéralogie des roches environnantes et avec celle des argiles d'Eybens, établissement de coupes en différentes localités de l'ancien lac...). Une synthèse en collaboration avec G. Monjuvent (Institut Dolomieu) doit être prochainement publiée.

\section{Analyse mécanique des conditions de glissement}

A partir de données physiques limitées, nous avons fait une tentative de reconstitution du mode de glissement. Nous avons établi un programme de calcul, adapté aux stabilités de pentes dans des massifs à litages horizontaux, qui pourrait constituer un mode de reconnaissance rapide des zones critiques dans des sites comparables.

\subsection{Propriétés mécaniques de l'argile varvée}

Le glissement s'est produit brusquement à une époque de forte humidité dans un massif saturé. On considérera donc la stabilité à court terme de la pente. Nous caractériserons le matériau par sa cohésion non drainée $C_{\mathrm{u}}\left(\varnothing_{\mathrm{u}}=0\right)$ à la teneur en eau de saturation $\left(w_{\text {sat }}=28,5 \%\right)$.

La cohésion non drainée a été déterminée à partir d'essais de cisaillement rapide (essai Casagrande) et nous avons pris en compte la résistance mécanique résiduelle au palier, étant donnés les grands déplacements à considérer le long de la surface de glissement. Ces essais ont été effectués sur des échantillons à section carrée de $100 \mathrm{~mm} \times 100 \mathrm{~mm}$ (vitesse de déplacement horizontal : $10 \mathrm{~mm} /$ minute) avec un bâti Casagrande classique, et sur des échantillons de largeur $250 \mathrm{~mm}$ et de longueur $400 \mathrm{~mm}$ (vitesse de déplacement horizontal : $20 \mathrm{~mm} /$ minute) avec un bâti spécialement conçu à I'I.R.I.G.M. pour des recherches sur le "rôle de l'échelle". Ce boîtier de grandes dimensions a permis d'obtenir des surfaces de glissement d'aspect beaucoup plus conforme aux observations faites in situ : présence de stries et autres 


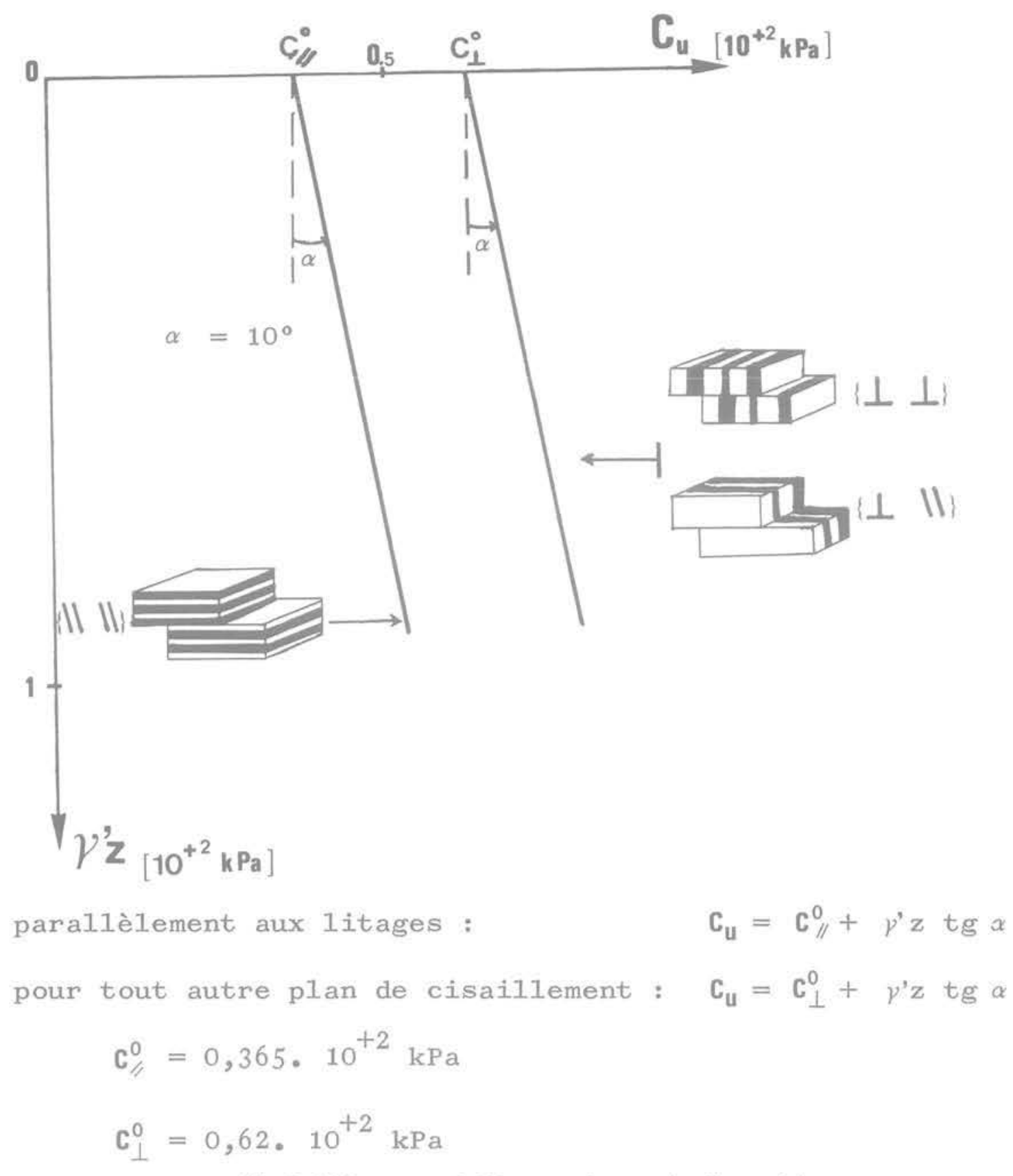

Fig. 5 Résistance au cisaillement anisotrope (argile varvée)

marqueurs de déplacement ( $u$ tectoglyphes " dus à des hétérogénéités locales), suivant le plan de glissement; formation d'un plan de cisaillement correct très difficile à engendrer dans un petit boîtier à cause du mouvement des blocs les uns par rapport aux autres, suivant le plan d'anisotropie.

De la série d'essais mécaniques effectués, on peut tirer les conclusions suivantes sur le massif d'argile varvée.

\section{* Homogénéité horizontale}

Le dépôt des couches d'argile s'étant effectué dans un lac de grandes dimensions et dans des conditions de sédimentation très calme, on peut compter sur une bonne homogénéité horizontale du matériau, malgré l'importance du glissement considéré (plus de $300 \mathrm{~m}$ de longueur pour le profil $A B$ ). Les essais mécaniques pour deux prélèvements (1 et 2 , fig. 3 ), l'un au centre de la zone déstabilisée (2), l'autre au niveau de la niche d'arrachement (1), le confirment d'ailleurs.
* Variation de la cohésion avec la profondeur

Les difficultés d'accès n'ont pas permis d'effectuer des prélèvements à forte profondeur, mais une étude comparative avec les résultats obtenus au «vane-test $\%$ sur le site analogue de Ponsonnas (même matériau, valeurs de la cohésion concordantes avec celles de Monestier du Percy) nous ont permis de fixer une loi de variation de la cohésion avec la hauteur de sédiments susjacents :

$$
\mathrm{C}_{\mathrm{u}}=\mathrm{C}_{\mathrm{o}}+\gamma^{\prime} \mathrm{z} \operatorname{tg} \alpha
$$

$z$ étant la profondeur à partir de la crête (point $\mathrm{A}: \mathrm{z}=0$, voir fig. 8).

\section{j Anisotropie discontinue}

L'essai Casagrande est le plus approprié pour un étude de la variation de la résistance au cisaillement avec l'orientation du plan de glissement, puisque dans cet essai, ce plan est imposé. 
Nous avons effectué des essais de cisaillement suivant les trois plans principaux : plan horizontal des litages et les deux plans perpendiculaires (fig, 5). La structure de l'argile varvée se traduit, au point de vue mécanique, par une anisotropie discontinue : les plans de litage représentent les seuls " plans de faiblesse ", la cohésion étant constante, à profondeur donnée pour toute autre orientation.

Les essais $|\perp / /|$ et $|\perp \perp|$ qui donnent la même résistance mécanique sont dorénavant groupés sous l'appellation $\mid \perp$ | et les essais $\mid / / /$ deviennent essais $|/|$. Dans les calculs on prendra:

$-\mathrm{C}_{\mathrm{u}}=\mathrm{C}_{\mathrm{u}}$ pour un plan de cisaillement horizontal;

$-C_{u}=C_{u}^{\perp}$ pour tout plan de cisaillement non horizontal.

\section{2 Étude de stabilité}

Nous avons effectué une étude de stabilité bidimensionnelle sur un profil "axial» du glissement, le profil $A B$ (fig. 3 et fig. 11), pour lequel nous disposons de renseignements suffisants (topographie avant et après glissement - profils sismiques - rotations du litage) et où les effets de bords (remontée en surface des terres noires) n'apparaîtront pas.

a) Indication de surface sur la zone en glissement

Soit A le sommet du profil considéré, correspondant à la niche d'arrachement, et B le pied du glissement correspondant à la présence de lames d'argile.

L'examen comparatif avec des photos aériennes (1970) permet d'évaluer le déplacement de certains points : le chemin avant glissement se trouvait en crête, un buisson situé dans la partie médiane du profil a subi un déplacement horizontal de soixante dix mètres environ...

La pente avant glissement peut être considérée comme uniforme $\left(\beta_{1}=11^{\circ} 6\right)$. On assimilera également pour le calcul la pente finale à une pente uniforme $\left(\beta_{t}=8^{\circ}\right)$. Ceci est en accord avec le remblaiement important en aval du pied B du glissement et le décalage d'une trentaine de mètres du cours du ruisseau (voir § 2.1).

b) Indication en profondeur sur la zone en glissement

Les profils sismiques II (suivant le profil $\mathrm{AB}$ ) et III (perpendiculairement au profil $A B$ ) permettent de caler les résultats théoriques en apportant une indication sur la profondeur de sol remanié (fig. 11).

Par ailleurs, le comportement anisotrope des argiles varvées suggère que le plan horizontal est une direction préférentielle de glissement. La méthode du cercle de glissement n'autorise pas la prise en compte d'une telle anisotropie. Nous supposerons donc une ligne de glissement polygonale avec mobilisation de la cohésion minimale $\mathrm{C}_{\mathrm{u}}$ (fonction de $\mathrm{z}$ ) suivant le plan horizontal, et une cohésion $\mathrm{C}_{u}^{+}$(fonction de $\mathrm{z}$ ) pour les plans de glissement inclinés (fig. 8).

Enfin, comme il s'agit d'un massif d'argile saturée en écoulement plastique, nous considérerons que le glissement se fait à volume constant (pas de foisonnement de la zone en mouvement).

\section{c) Cinématique du glissement}

L'ensemble des indications précédentes nous permet de supposer la cinématique suivante: la surface de glissement est polygonale avec un plan en pied tangent à la lame d'argile émergente (à $\left.30^{\circ}\right)$, et avec dans sa partie médiane, un plan parallèle aux litages horizontaux. Le glissement s'est traduit (fig. 6 et fig, 11), dans la partie amont du profil par un effondrement avec distorsions locales (litages à $15^{\circ}$ en crête et surface du chemin basculée de $30^{\circ}$ ), et dans la partie avale par une translation horizontale non uniforme (fissures de traction nombreuses en surface). Cette translation est due au recul du pied $B$ du glissement (translation $\Delta \mathrm{L}$ ), mettant en butée le massif de sol aval et décalant le cours du ruisseau.

d) Détermination de la surface de glissement initiale

L'originalité de cette étude de stabilité est de permettre de retrouver la géométrie avant glissement du massif de sol instable. Cette démarche a deux avantages:

- ll est évidemment plus logique d'étudier l'instabilité du massif juste avant glissement (suivant ce que nous appellerons la surface de glissement initiale) plutôt qu'après glissement (suivant l'état final de la surface de glissement ou surface de glissement finale). Ces deux surfaces se différencient dans la partie basse du glissement.

- Il est intéressant d'obtenir une estimation des déplacements $(\Delta \mathrm{L}, \Delta \mathrm{H})$ en pied du glissement.

La surface de glissement finale est déterminée, une fois connus les paramètres géométriques $\left(D_{t}, \theta\right)$ (fig. 6). Le calcul de stabilité du paragraphe suivant va nous permettre d'en trouver les valeurs critiques.

La surface de glissement initiale (avant écoulement) correspondant à ces valeurs est reconstituée en écrivant la non-variation de volume du massif de sol en mouvement (fig. 7). On en déduit par ailleurs les déplacements $\Delta \mathrm{L}$ et $\Delta \mathrm{H}$ en pied de glissement.

e) Modalités du calcul

Nous appliquons la méthode des blocs avec résultante nulle au massif de sol "initial " avant glissement (fig. 8). Le coefficient de sécurité $F$ est défini par:

$$
C_{u} \text { mobilisée }=\frac{C_{u}}{F} \text {. }
$$

Pour chaque élément plan de la surface de glissement, on prend en compte la cohésion $\mathrm{C}_{4}$ correspondant à la profondeur z moyenne de ce plan (fig. 8).

Pour chaque géométrie supposée de la surface de glissement finale $\left(D_{f}, \theta\right.$ fixées), on détermine la surface de glissement initiale associée $(\Delta \mathrm{H}, \Delta \mathrm{L})$ et le coefficient de sécurité correspondant (F).

\section{f) Résultats}

Quelle que soit la profondeur $D_{f}, F$ passe par un minimum pour $\theta=\theta$ critique $\approx 38^{\circ}$ (fig. 9 ).

Pour $\theta=\theta$ critique, $F$ diminue continument avec la profondeur (fig. 10). On retrouve un résultat classique pour les sols cohérents purs : lorsque la résistance au cisaillement augmente peu avec la profondeur, on obtient toujours une rupture en profondeur.

g) Estimation de la surface de glissement réelle

Le coefficient de sécurité $F=1$ est atteint à partir d'une profondeur $D_{1}=17 \mathrm{~m}$. Si l'on admet comme acceptables les hypothèses à la base de ce calcul, on admet qu'il s'agit de la profondeur de glissement réelle. Ceci rend vraisemblable la présence des terres noires à cette profondeur ou du moins la présence d'un substratum plus consistant empêchant une rupture plus profonde. De plus, notons que $D_{f}=17 \mathrm{~m}$ est en accord avec les profils sismiques (fig. 4 et 11) et que le déplacement en pied correspondant $(\Delta H=-0,5 \mathrm{~m}$; $\Delta \mathrm{L}=+27,5 \mathrm{~m}$ ) (fig. 10) est compatible avec les déplacements des points repères évalués dans le profil $A B$. 


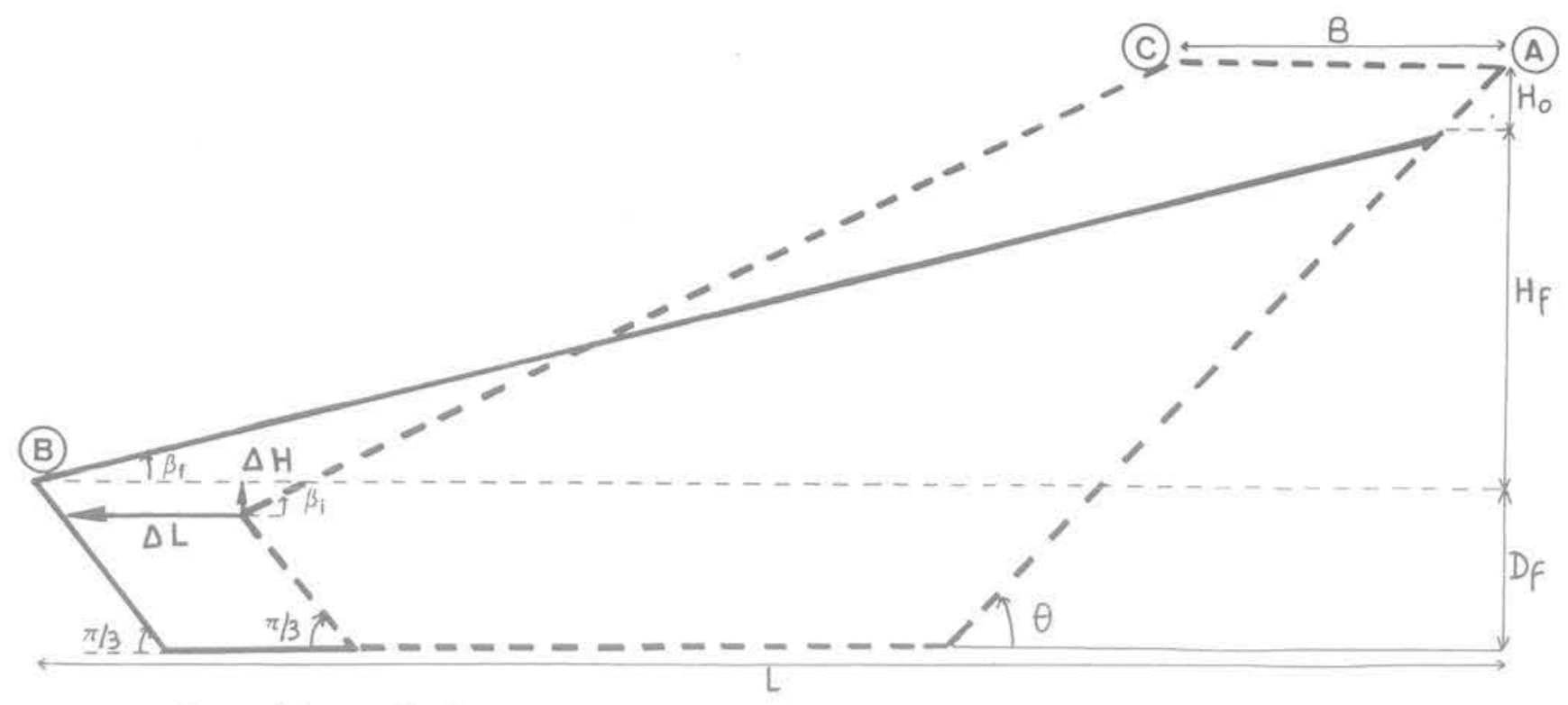

Paramètres fixés :

$\mathrm{L}=229 \mathrm{~m} \quad \mathrm{~B}=31 \mathrm{~m} \quad \mathrm{H}_{\mathrm{f}}+\mathrm{H}_{\mathrm{o}}=35,5 \mathrm{~m} \quad \beta_{i}=11^{\circ}, 6 \quad \beta_{f}=8^{\circ}$

$X_{\text {sat }}=2000 \mathrm{daN} / \mathrm{m} 3$

L'hypothèse de non variation de volume du massif de sol en glissement permet de déterminer

$$
\begin{aligned}
& \Delta H \quad f\left(D_{f}, \Theta\right) \\
& \Delta L \quad f\left(D_{f}, \Theta\right)
\end{aligned}
$$

- - - surface de glissement initiale (avant glissement)

surface de glissement finale (après glissement)

Fig. 6 Non variation de volume du massif de sol en glissement

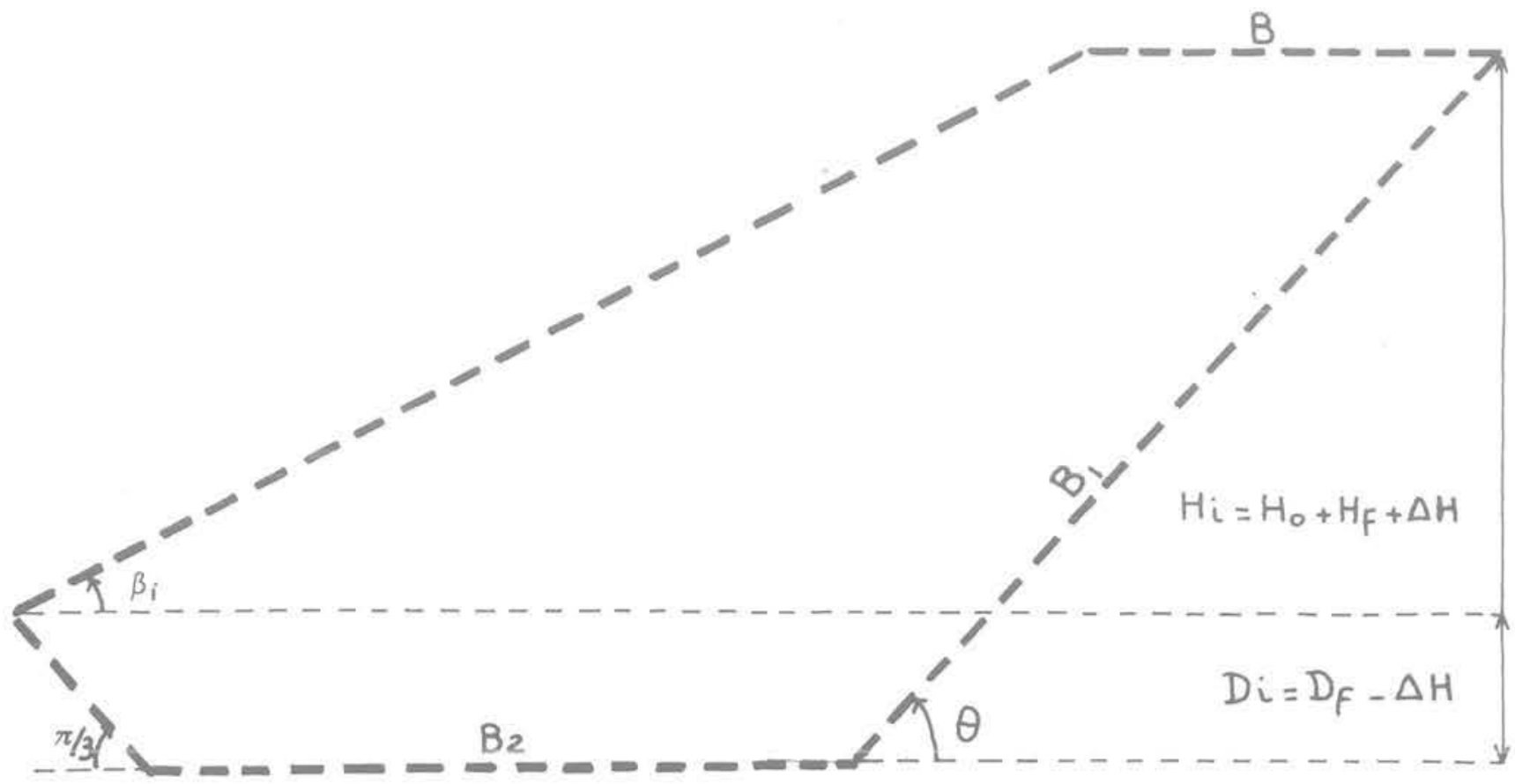

Fig. 7 Dimensions du massif de sol avant glissement (pour $D_{f}, \Theta$ fixés) 


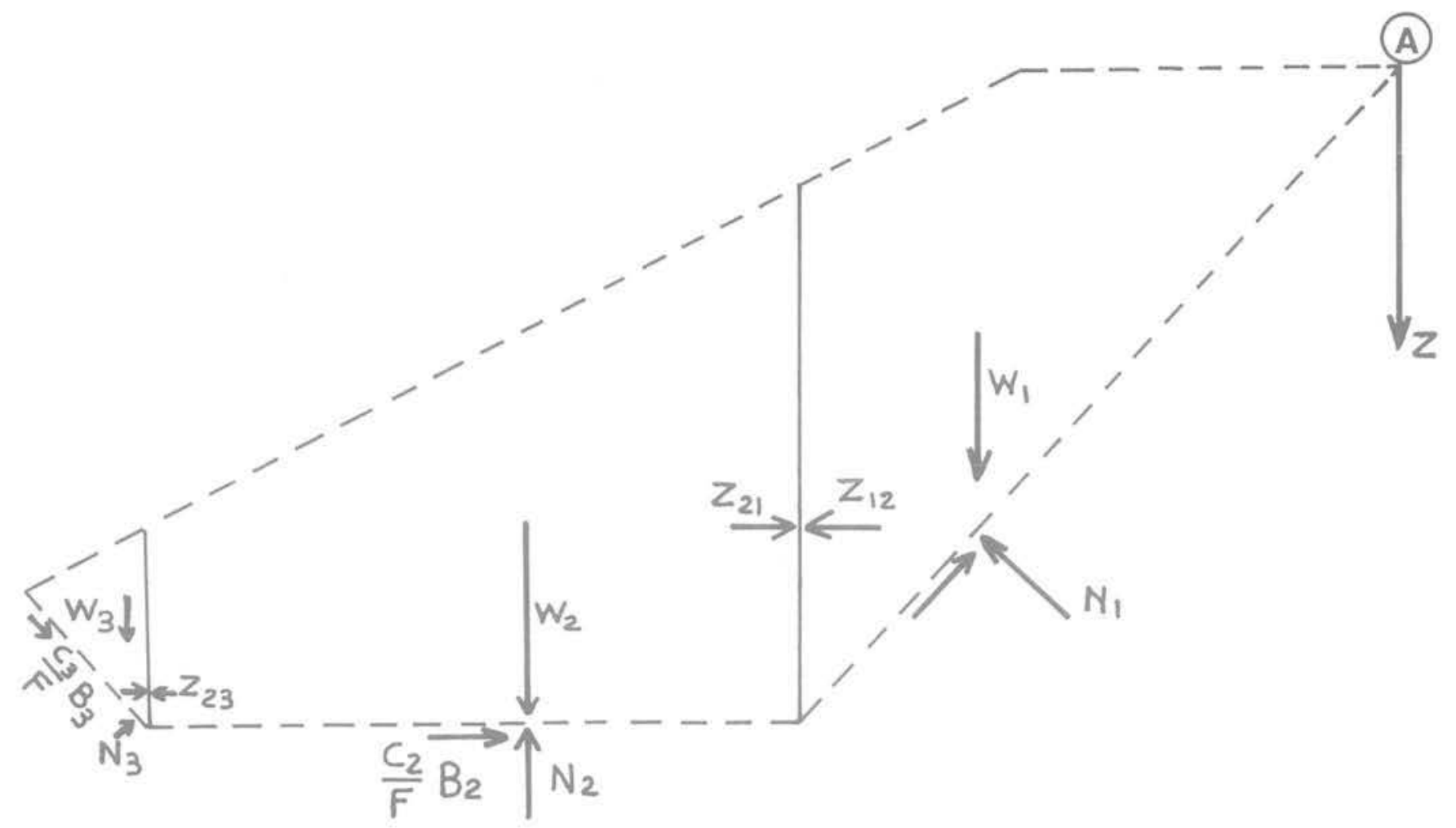

Méthode de la résultante nulle :

- Equilibre des forces pour chacun des trois blocs

- Efforts inter-blocs supposés horizontaux

- Cohésion non drainée prise en compte :

fonction de $1^{\prime}$ orientation de $1 \mathrm{a}$ base du bloc considéré

fonction de la profondeur moyenne de $1 \mathrm{a}$ base de ce même bloc $\mathrm{C}_{1}=\mathrm{C}_{\perp}^{0}+\gamma^{\prime} \mathrm{z}_{1} \operatorname{tg} \alpha=\mathrm{C}_{\perp}^{0}+\gamma^{\prime} \cdot\left[\frac{\mathrm{D}_{\mathbf{i}}+\mathrm{H}_{\mathbf{i}}}{2}\right] \cdot \operatorname{tg} \alpha$

$\mathrm{C}_{2}=\mathrm{C}_{1 / 2}^{0}+\gamma^{\prime} \mathrm{z}_{2} \operatorname{tg} \alpha=\mathrm{C}_{/ 1}^{0}+\gamma^{1} \cdot\left[\mathrm{D}_{i}+\mathrm{H}_{i}\right] \cdot \operatorname{tg} \alpha$

$\mathrm{C}_{3}=\mathrm{C}_{\perp}+\gamma^{\prime} \mathrm{z}_{3} \operatorname{tg} \alpha=\mathrm{C}_{\perp}+\gamma^{\mathrm{t}} \cdot\left[\frac{\mathrm{D}_{\mathrm{i}}^{\mathrm{i}}}{2}+\mathrm{H}_{i}\right] \cdot \operatorname{tg} \alpha$

Fig. 8 Détermination du coefficient de sécurité

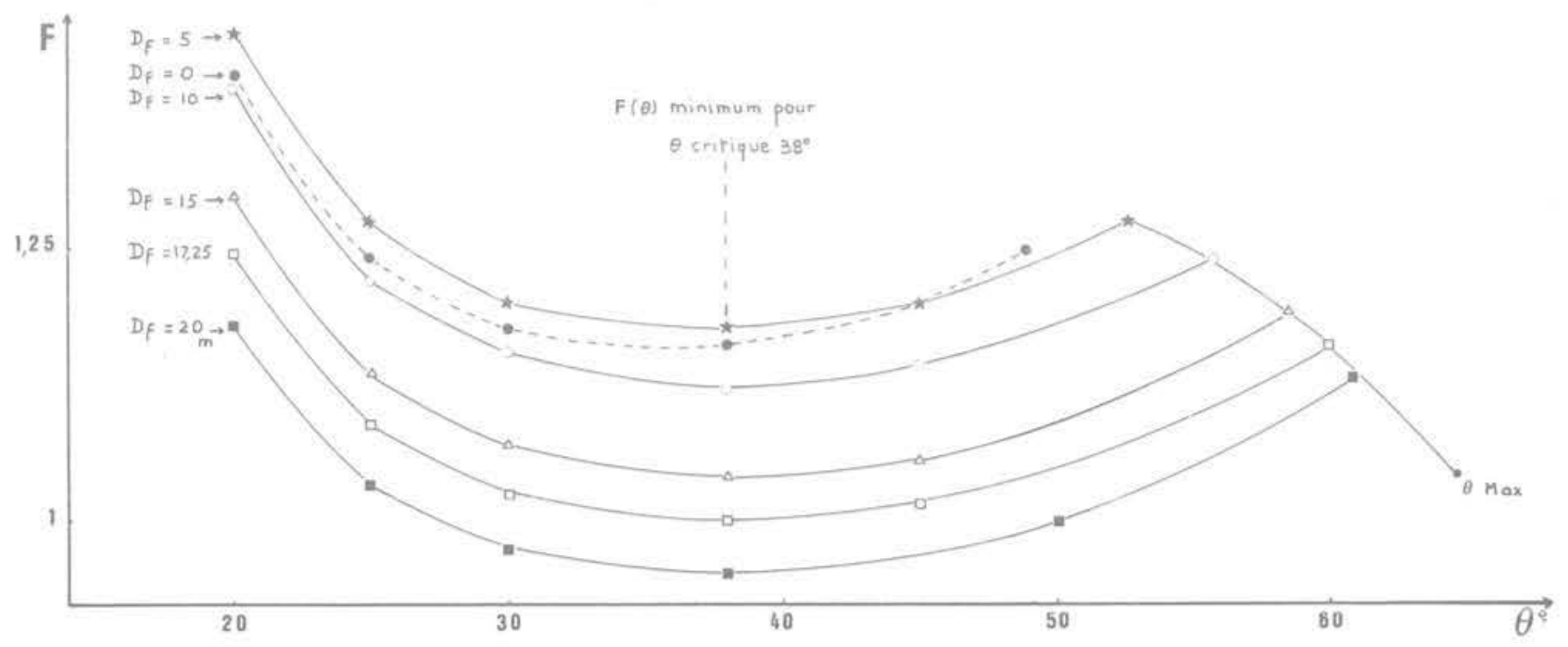

Pour $\theta=\theta$ max, $1 \mathrm{a}$ paroi verticale inter-blocs 1 et 2 passe par le point $C$

Fig. 9 Variation de $F$ en fonction de $D_{f}$ et $\Theta$ 


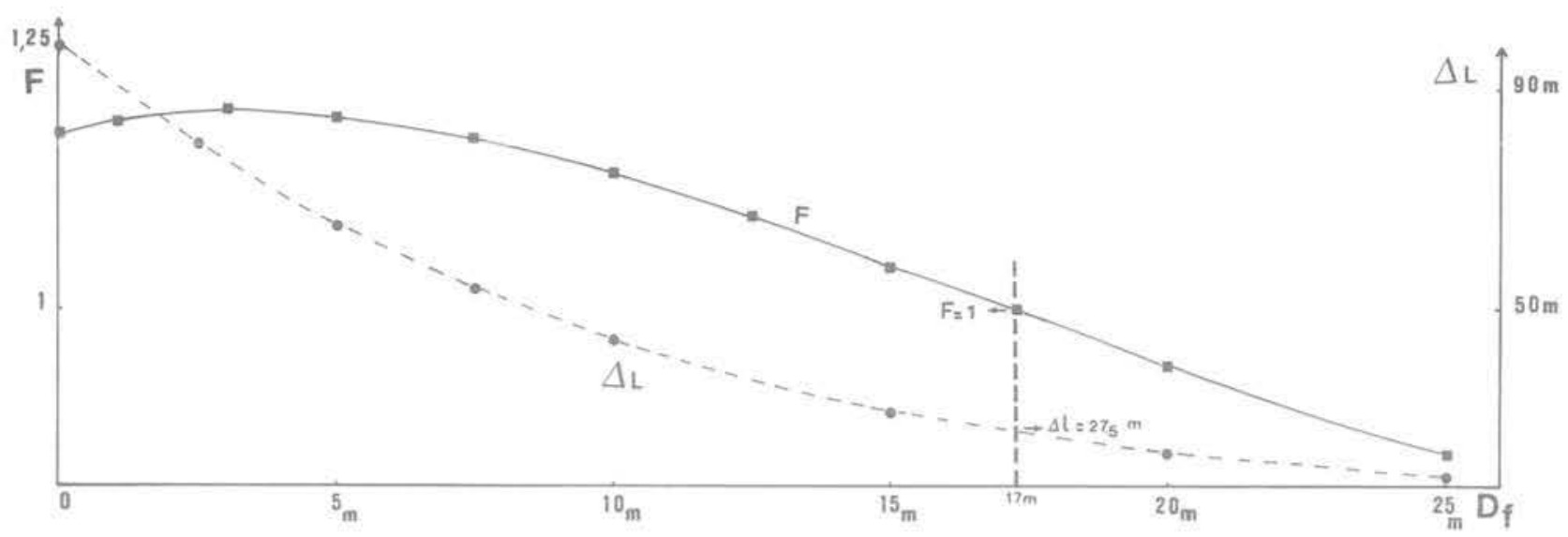

Fig. 10 Variation de $F$ et $\Delta L$ en fonction de $D_{f}^{\prime}$ pour $\Theta=\Theta$ critique $=38^{\circ}$
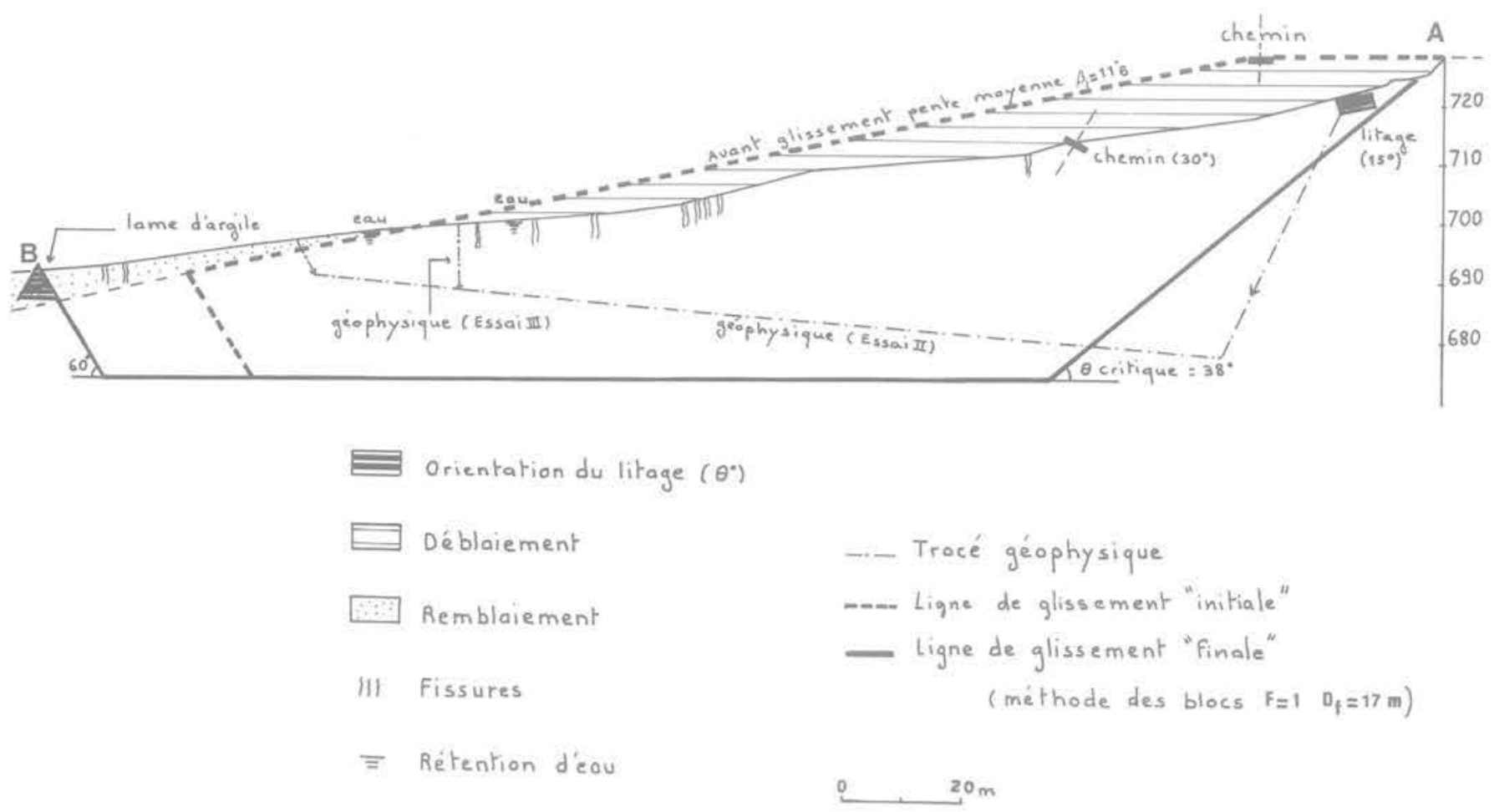

Fig. 11 Profil "axial» du glissement : observations, mesures et résultats de l'étude de stabilité

\section{Solutions envisageables et implications administratives}

II était financièrement exclu, étant données l'ampleur du glissement et la valeur des biens effectués, d'entreprendre une remise en état des terrains glissés pour les rendre à leur vocation première. II importait donc de proposer des solutions simples pour empêcher l'extension du mouvement el restaurer les terrains glissés en leur trouvant une nouvelle vocation.

Cette tâche incomba à la Direction Départementale de l'Agriculture.

\subsection{Mesures immédiates}

- Les premiers travaux envisageables devaient concerner l'assainissement des zones marécageuses situées à l'amont du glissement pour diminuer les possibilités d'infiltration des eaux vers la couche d'argile varvée.

- Par ailleurs, il fallait consolider un petit barrage situé en aval du glissement qui avait été endommagé par les remous du torrent et les coulées boueuses consécutives au glissement. Enfin, le chemin communal traversant le glissement devait être rétabli. 
Pour ces travaux de drainage, de reprofilage du ruisseau, de consolidation du seuil et d'aménagement d'un nouveau chemin (devis global $200 \mathrm{kF}$ ) une subvention à $75 \%$ du Département put être obtenue rapidement.

Simultanément aux travaux engagés, le Maire présenta, par l'intermédiaire de la D.D.A. un dossier au Comité Départemental d'Expertise des Calamités Agricoles. Bien que ce type de dégradation ne soit pas, en général, pris en compte par le Comité, l'importance des dégâts incitait à ouvrir un dossier. En effet, au cours de ces quatre jours, 9 ha de pâturages et de terres cultivables avaient été emportés et devenaient inutilisables. Étaient donc concernés par ce sinistre les propriétaires et les exploitants, puisque la plupart de ces terres étaient louées à des éleveurs.

Après examen, le sinistre fut retenu comme calamité agricole par arrêté interministériel, pris par les ministres de l'Agriculture et de l'Économie, en date du 14 juin 1979. L'indemnisation des propriétaires et des exploitants par le Fonds de Garantie des Calamités Agricoles était dès lors possible.

\subsection{Recherche d'une solution pour le reclasse- ment des terrains glissés}

A plus long terme se pose la question du devenir des surfaces glissées. Du point de vue économique et même technique, les observations faites sur l'importance du glissement (forte épaisseur de la zone remaniée, désorganisation complète de la topographie et du drainage naturel...) ne permettent pas d'espérer recréer des cultures, ni même des pâturages.

Pour ce type de terrain, une solution paraît être l'achat par l'État en vue de la création d'une série domaniale de Restauration des Terrains en Montagne (série R.T.M.). Elle présente l'avantage de soulager la commune des dépenses nécessaires pour assurer une certaine sécurité sur les 9 ha du glissement. Les opérations à mener dès lors par l'État sont, dans l'ordre, le drainage, la correction torrentielle, le réembroussaillement puis le reboisement des terres.

\subsection{Quelques réflexions}

Le glissement de terrain de Monestier du Percy est un bon exemple de dégradation par l'érosion d'une zone fragile et peu peuplée du territoire national.

En dehors des conditions climatiques particulières, agissant le plus souvent comme élément aggravateur et déclencheur d'un phénomène déjà préprogrammé au niveau du terrain, on peut incriminer avant tout l'exode rural et la perte de main d'œuvre et de savoir-faire local. Les anciens connaissaient les techniques et l'intérêt de l'entretien des moindres éléments du réseau hydrographique naturel et artificiel!

On remarque une certaine prise de conscience des citoyens vis-à-vis de l'importance des facteurs déterminant notre environnement, mais paradoxalement, ceci se traduit par un transfert progressif de responsabilité sur les collectivités locales, puis sur l'Administration et l'État.

Au niveau des moyens mis en œuvre pour restaurer les terrains après dégradation naturelle, on doit noter enfin la faiblesse des budgets communaux et le relais indispensable des budgets départementaux, régionaux ou nationaux.

On peut penser raisonnablement que, selon le viel adage « mieux vaut prévenir que guérir ", l'action des collectivités locales et des services techniques départementaux pourrait être renforcée par la création d'un corps de surveillance et d'entretien des terres comme if en existe pour les routes et au moins partiellement pour les avalanches (observations nivométéorologiques et relevés topographiques effectués par les agents forestiers).

\section{Conclusion}

Les méthodes d'analyse des mouvements de terrain sont aujourd'hui bien au point. Mais pour aboutir, à partir de l'interprétation des phénomènes passés, à la prévision des mouvements ultérieurs et à l'évaluation de la sécurité des pentes naturelles, de nombreuses études "géomécaniques" seront encore nécessaire. La mise au point d'un programme de calcul adapté à un type de mouvement et à un matériau bien identifié passe par un étalonnage sur des mouvements réels comme celui que nous avons présenté dans cette étude pluridisciplinaire.

La collaboration engagée sur les glissement dans les argiles litées du Trièves, entre géologues et mécaniciens des sols d'un laboratoire universitaire et les services techniques départementaux (D. D. A., D. D. E.) doit être poursuivie. Ce type d'étude s'inscrit en complément logique de l'inventaire et de la cartographie systématique des mouvements de terrain réalisés à d'autres échelles au plan départemental (cartes communales de risques naturels) et au plan national (cartes Zermos).

\section{Références bibliographiques}

Antoine P. (1977). Réflexions sur la cartographie Zermos et bilan des expériences en cours. Bull. B. R. G. M., $2^{\circ}$ série, section III, n० $1 / 2$, p. 9-20.

Antoine P., Biarez J., Desvarreux P. et Mougin J.P. (1971). Les problèmes posés par la stabilité des pentes dans les régions montagneuses. Géologie alpine, t. 47, p. 5-24.

Gourc J.P. (1978). Cours de Stabilité des pentes. École Nationale des Travaux Publics de l'État, Lyon, $141 \mathrm{P}$.

Huff W.D. (1974). Mineralogy and provenance of pleistocene lake clay in an alpine region. Geol. Soc. of Amer. Bull., vol. 85; p. 1455-1460.

Koseoglu M. (1977). Étude géologique et géotechnique routière (route nationale 75 entre Vif et le col de la Croix Haute), thèse Docteur Ingénieur, Grenoble, $228 \mathrm{P}$.

Monjuvent G. (1973). La transfluence Durance-Isère. Essai de synthèse du Quaternaire du bassin du Drac (Alpes françaises).

Géologie alpine t. 49 , p. 57-118.

Sultan H.A. et Seed B. (1967). Stability of sloping core earth dams. Journal of the Soil Mechanics and Foundations Division, Proceeding of the American Society of Civil Engineers, SM4 p. 45.

Tachker Y. (1973). Méthodes utilisées dans le Département de l'Isère pour la délimitation des zones exposées à des risques naturels. Symposium Sol et Sous-sol et sécurité des Constructions, Cannes, 1, p. 377-388. 